REVISTA ANDALUZA DE ANTROPOLOGÍA.

NÚMERO 8: TURISMO DE BASE LOCAL EN LA GLOBALIZACIÓN

MARZO DE 2015

ISSN 2174-6796

[pp. 155-159]

http://dx.doi.org/10.12795/RAA.2015.i08.10

\title{
PEDREÑO CÁNOVAS, ANDRÉS (coord.) (2014). De cadenas, migrantes y jornaleros. Los territorios rurales en las cadenas globales agroalimentarias. Madrid: Talasa, $236 \mathrm{pp}$.
}

\section{Salvador Cayuela Sánchez}

Universidad de Murcia

Los franceses Luc Boltanski y Ėve Chiapello argumentaban hace ya algunos años en su libro El nuevo espíritu del capitalismo, que este sistema económico había sido capaz de amoldar las reivindicaciones laborales del Mayo del 68 francés a su propia estructura, funcionamiento y necesidades. En este sentido, como una especie de burbuja extremadamente maleable pero al tiempo irrompible, parecía que hubiese asimilado las críticas sesentayochistas al aburrido trabajo rutinario tan propio del fordismo, ordenando un nuevo paradigma laboral dirigido por la flexibilidad, la movilidad y la creatividad. Ahora bien, este paradigma laboral, instalado ya en mayor o menor medida en prácticamente todos los países del globo, no iba a tener las mismas consecuencias e implicaciones para todos los segmentos sociales. En efecto, mientras unos iban a poder realizarse plenamente dentro de estos nuevos parámetros como auténticos empresarios de sí -por utilizar la jerga anglofoucaultiana-, otros lo iban a sentir en sus cuerpos y en sus mentes como un auténtico riesgo permanente -con Ulrich Beck-, incluso como un movimiento corrosivo del carácter -adoptando la exitosa expresión de Richard Sennett-. El compendio que aquí se reseña, coordinado por Andrés Pedreño Cánovas, y titulado De cadenas, migrantes y jornaleros, puede ser entendido en este marco interpretativo como un esfuerzo colectivo por comprender y analizar las lógicas de estas nuevas formas de 
trabajo, esta vez en el sector agrícola, presentes en diversos escenarios locales, y conectadas a un tiempo con largas cadenas globales agroalimentarias -como acertadamente destaca el subtítulo, Los territorios rurales en las cadenas globales agroalimentarias-. Pero además se trata, en efecto, de cadenas por las que transitan mucho más que flujos de dinero y mercancías: a saber, personas, ahora flexibles y móviles, llamadas y utilizadas en enclaves agrícolas que condensan, a modo de nudos inesperadamente fáciles de clonar, dinámicas productivas postmodernas.

De hecho, ya al ojear las primeras páginas del compendio, se nos advierte sobre los cinco ejes conceptuales que ordenan los espacios discursivos en los que se inscriben los diversos capítulos, en una edición por lo demás muy cuidada y agradable. Así, cabría distinguir según el propio coordinador, un primer eje conceptual en el que se reunirían los capítulos 1 -escrito por el propio Pedreño Cánovas- y 2 -firmado por Norma Steimbreger-, sobre las Cadenas globales, territorios de producción y estrategias empresariales. Dentro de este eje argumental, Andrés Pedreño analiza la reconfiguración en la Región de Murcia (España) del sector agroindutrial, ya de cierta tradición en la zona, pero que ahora parece apostar por el cultivo de la uva de mesa, pensada como un auténtico maná supuestamente revolucionario para la región. En este capítulo, titulado "Encadenados a fetiches. Del enfoque de las cadenas de mercancías a la sostenibilidad social de los enclaves de producción de la 'uva global"', centra su atención Pedreño en este producto emblemático del sureste español, analizando las transformaciones derivadas de la "creación" de nuevas variedades de uva de mesa, y la configuración aparejada de grandes empresas y cooperativas dedicadas exclusivamente a esta fruta. En un contexto en el que los inmigrantes y las mujeres locales han conformado un auténtico "ejército de reserva" condición indispensable para mantener las tasas de beneficio de la nueva agricultura-, se habría producido -según Pedreño- una "destrucción creativa del territorio", donde dos esferas extracapitalistas -la propia uva de mesa autóctona y los pequeños agricultores autónomos-, habrían sido absorbidas por la nueva industria agroalimentaria.

Un segundo capítulo -aún dentro de este primer eje y titulado "Dinámica del capital global en el control territorial de una cadena agrícola. El caso de la fruticultura del norte de la Patagonia (Argentina)"-, Norma Graciela Steimbreger expone un estudio de caso encargado de mostrar el alcance e intensidad de esa movilidad adquirida en nuestros tiempos por un "capital global", emanado no obstante de estrategias de acumulación empresarial íntimamente conectadas con relaciones de poder insertas a nivel local y regional. Así, y partiendo del caso del sector frutícola del norte de la región argentina de Patagonia, analiza Steimbreger cómo las grandes corporaciones comercializadoras y distribuidoras de productos frescos son capaces, por un lado, de orientar en gran medida las dinámicas de los mercados agroalimentarios mundiales y, por otro lado, de configurar una gradación de los territorios productivos locales, clasificándolos según su valoración desigual. 
Saltando a un segundo eje conceptual -que el coordinador del compendio titula Reclutamiento de fuerza de trabajo y composición social de los "nuevos jornaleros": mujeres $y$ colectivos migrantes-, encontramos el capítulo escrito por Alicia Regada, y titulado "Los 'nuevos jornaleros.' Construcción y fragmentación social de la fuerza de trabajo en los enclaves agrícolas globales". En este capítulo -el 6 según el índice-, Reigada aborda la composición social de un nuevo grupo de jornaleros inserto en los enclaves agrícolas globales, y configurado sobre la base de varios procesos recurrentes en distintas partes del globo. Así, en la formación de estos "nuevos jornaleros" nómadas y flexibles de los campos agrícolas globalizados, dinámicas como la etnización y feminización de la fuerza trabajo en las labores intensivas exigidas por los nuevos modelos productivos, la creciente flexibilidad y precariedad laboral, o la diversificación de las modalidades de "reclutamiento" de los trabajadores, serían fácilmente detectables en prácticamente cualquier lugar del mundo.

En un tercer eje conceptual, dedicado a Las condiciones de trabajo y los procesos de control de la producción, encontraríamos tres capítulos, el primero de los cuales correspondería al elaborado por Carlos de Castro, y titulado "La desdemocratización de las relaciones laborales en los enclaves globales de producción agrícola”. En este capítulo, Carlos de Castro expone cómo los nuevos enclaves globales de producción agrícola estarían conformando una determinada forma de entender el empleo, una "norma" que a su vez estaría erosionando el tradicional vínculo moderno entre trabajo y ciudadanía. Para sostener estas tesis, el autor analiza las formas de trabajo desarrolladas en varios enclaves de producción agrícola en España y América Latina, donde -de nuevo- pueden advertirse ciertas regularidades: una alta temporalidad en el trabajo; un elevado índice de trabajo informal; salarios bajos; ausencia de negociación colectiva; socavamiento de las condiciones de reproducción social de los trabajadores; etc. También en esta línea argumental, Alberto Riella, Mauricio Tubío y Rosario Lombardo participan en el compendio con su artículo "Los jornaleros de las cadenas globales de producción de alimentos en fresco: el caso del arándano en Uruguay”. Así, y tomando un estudio de caso preciso -como anticipa por lo demás el título-, los autores exponen cómo se habría conformado en Uruguay un enclave agrícola global en torno a la producción del arándano, poniéndolo en relación con procesos similares acaecidos en otros lugares del planeta. Finalmente, Germán Quaranta concluiría este tercer eje con su trabajo "La conformación de un mercado de trabajo transitorio migrante en un nuevo territorio productivo: el caso de la olivicultura, Pomán, Catamarca, Argentina”. En este artículo, Quaranta analiza la configuración de un mercado laboral adaptado a las exigencias de las empresas agrícolas capitalistas, exigidas en su modo de producción de ingentes cantidades de trabajadores en momentos muy puntuales del ciclo productivo. Así, partiendo de la conformación del nuevo espacio productivo olivícola desarrollado en el departamento de Pomán, Catamarca, en Argentina, el autor analiza las estructuras y dinámicas de una 
particular organización laboral, así como las estrategias empresariales de reclutamiento de mano de obra, conducentes en gran medida a la creación de un mercado de trabajo transitorio y migrante.

Un cuarto eje conceptual que ordena este compendio se correspondería con Las estrategias de reproducción social y de socialidad de los trabajadores, y aglutinaría a su vez a otros tres capítulos. El primero de ellos -el capítulo 7 del índice- sería el presentado por Elena Gadea, Antonio J. Ramírez y Joaquín Sánchez, y titulado "Estrategias de reproducción social y circulaciones migratorias de los trabajadores en los enclaves globales". Este trabajo estaría destinado a mostrar cómo los procesos actuales de reestructuración del sistema agroalimentario habrían incidido tanto en los perfiles como en las formas tradicionales de movilidad de los jornaleros. Al tiempo, la configuración de estos nuevos escenarios productivos agroalimentarios habrían ocasionado profundas transformaciones tanto en las condiciones de vida de los trabajadores, como en los propios mecanismos de cohesión social de esos nuevos territorios productivos. Todo ello permitiría a los autores esbozar las características idiosincrásicas presentes en los "territorios de la globalización agroalimentaria”. Un segundo capítulo de este eje -el $8^{\circ}$ del libro-, firmado por Sara María Lara, Kim Sánchez y Adriana Saldaña, y titulado "Asentamientos de trabajadores migrantes en torno a enclaves de agricultura intensiva en México: nuevas formas de apropiación de espacios en disputa", establece una comparación analítica entre dos regiones mexicanas de agricultura intensiva: una en el Estado de Morelos, y otra en el Estado de Sinaloa. Así, en ambos enclaves, la actividad agrícola habría supuesto una considerable concentración de capital y recursos naturales, pero estructurados sobre la base de dos estrategias bien distintas: la extendida en Morelos, en manos de pequeños productores dedicados al cultivo de hortalizas, y cuya producción estaría destinada principalmente al abastecimiento de la Ciudad de México. Y la desarrollada en Sinaloa, una agricultura intensiva copada por grandes empresas hipermodernas dedicadas a una producción hortícola de exportación. Con todo, ambos modelos habrían generado dinámicos mercados de trabajo capaces de articular complejos circuitos de migración de trabajadores desde el sur del país. El tercer y último capítulo de este eje, aportado por Mónica Bendini y titulado "La migración estacional de trabajadores agrícolas: un tema preeminente y complejo", seguiría con estas problemáticas, centrando su atención en la propia naturaleza estacional en el agro, y dirigiendo sus reflexiones a la región del norte de Patagonia. Partiendo así de este caso particular, analiza ciertas dinámicas migratorias de sustitución, donde unos trabajadores ocupan los puestos que otros han abandonado en su búsqueda por mejores salarios y condiciones de trabajo. Sobre estos procesos continúa elaborando un mapa donde se muestran determinadas relaciones formales e informales en el acceso al trabajo, y donde nuevos "intermediarios" juegan un papel crucial. 
Un quinto y último eje conceptual aglutina los dos capítulos destinados a cerrar el compendio, orientados sobre la temática Nuevas formas públicas y privadas de regulación transnacional de las relaciones laborales. Así, el primero de estos capítulos, presentado por Natalia Moraes e Isabel Cutillas y titulado "Nuevos dispositivos de regulación transnacional: un análisis sobre los estándares de calidad y responsabilidad social y su impacto en los enclaves globales agrícolas", pretende analizar varios dispositivos a través de los cuales se advierte esa red transnacional de regulación sobre la que se articulan las nuevas tecnologías normativas. En este sentido, los estándares de calidad y responsabilidad social empresarial son entendidos como nuevos dispositivos de regulación, asignados ahora a empresas privadas encargadas de certificar tanto la calidad de los productos, como las buenas prácticas agrícolas y la responsabilidad social de las empresas. De este modo, los mecanismos y empresas de certificación habrían implantado una normalización privada de los productos agrícolas y sus formas de producción, en una dinámica global que tendría fuertes impactos en los enclaves agrícolas locales. Un último capítulo del compendio, firmado por Josefa Salete Barbosa Cavalcanti y titulado "De los extremos de la calidad y la permanente vulnerabilidad: los trabajadores del Vale de Sao Francisco, en el nordeste de Brasil", profundiza en la cuestión de cómo las actuales fases de desarrollo de la globalización económica provocan un incremento considerable de las desigualdades observadas en los enclaves locales. Así, tomando como campo de análisis esta región del nordeste de Brasil, se analizan las condiciones de trabajo de los migrantes empleados en el sector hortofrutícola local -conectado claro está con cadenas agroalimentarias globales-, expuestos a un tiempo a las condiciones laborales propias del sector en los tiempos actuales, pero también a los protocolos y normas de buenas prácticas agrícolas exigidos por las agencias privadas de certificación.

Se trata, en definitiva, de un compendio preocupado por las nuevas formas de trabajo emergidas en el actual contexto de globalización económica, centrado en el sector agrícola pero que apunta a dinámicas desafortunadamente extrapolables a casi cualquier sector económico. En este sentido, aspectos como las nuevas formas privadas de normalización, la desdemocratización de los colectivos de trabajadores, o la aparentemente imparable conquista de las esferas extracapitalistas por el sistema económico dominante son, en efecto, fácilmente detectables en prácticamente todos los lugares del globo. Por todo ello, y en nuestra opinión, se trata de una lectura obligada -aunque placentera- para todo aquel interesado en el mundo del trabajo hoy, y en los procesos "glocales" en los que se inserta. 